\title{
Procesos de investigación-acción en aprendizaje y servicio solidario: percepciones de los protagonistas pedagógicos en el proyecto Hiri Lagunkoiak
}

\section{Ángela García Pérez}

Fundación Zerbikas y Universidad de Deusto, España Aurelio Villa Sánchez

Fundación Horreum y Universidad de Deusto, España

\section{Aitziber Mugarra Elorriaga}

Fundación Zerbikas y Universidad de Deusto, España

\section{Resumen}

Ciudades Amigables para todas las personas: investigación-acción en accesibilidad urbana a través de OpenStreetMap ${ }^{i}$ es un proyecto de aprendizaje y servicio solidario para fomentar la conciencia cívica en el derecho de accesibilidad, protagonizado ya por más de 1000 adolescentes y/o personas con movilidad reducida en el País Vasco. En este artículo compartimos un análisis cualitativo de sus percepciones, desde su rol investigador en su compromiso por la transformación del entorno. Dados los resultados ofrecidos en términos de necesidad, de aprendizaje y de servicio, proponemos una síntesis de interacción entre el continuum de percepción de las propias emociones-valores-compromiso y su orientación a la acción, en la cogeneración de una ciudadanía competente y solidaria.

\section{Palabras clave}

Aprendizaje y servicio solidario, ciudades amigables, investigación-acción, derechos humanos, percepciones. Iberoamericana de Aprendizaje Servicio, 5, 62-86. DOI10.1344/RIDAS2018.5.5 


\title{
Action-research processes in service learning: perceptions of pedagogical protagonists in Friendly Cities 4All project
}

\begin{abstract}
Friendly Cities for everyone (4All): action-research in urban accessibility through OpenStreetMap is a service learning project to promote civic awareness in the right of accessibility, already carried out by more than 1000 teenagers and/or people with reduced mobility in The Basque Country. In this article we share a qualitative analysis of their perceptions, from their research role in their commitment to the transformation of the environment. Given the results offered in relation to social challenge, learning and service, we propose a synthesis of interaction between the continuum of perception of their own emotions-values-engagement and their orientation to action, in the co-generation of a competent and inclusive citizenship.
\end{abstract}

\section{Keywords}

Service Learning, friendly cities, action research, human rights, perceptions. 


\section{Introducción}

La ciudad deviene educadora cuando asume intencionalidad y responsabilidad en la formación y desarrollo de todos sus habitantes. Desde esta concepción, se entiende que la función educadora no recae únicamente en los centros educativos formales, sino que éstos resultan un parte más de la compleja red educativa de la ciudad.

Campo, 2015, 162.

Más allá de los movimientos actuales de Ciudades Educadoras, Ciudades Accesibles o Ciudades Amigables (propios de la última o dos últimas décadas) podríamos remontarnos a una ciudadanía democrática, una gran comunidad cuidadora y educativa-experiencial argumentada por John Dewey en los inicios del siglo $X X$ y a las referencias en su construcción que el propio filósofo-pedagógo encontró en civilizaciones que nos han precedido.

Términos como Innovación Social, o incluso Educacionalización (respuesta desde el ámbito educativo formal a retos que no han sido considerados propiamente como educativos) son novedosos, pero su fundamento y recorrido en la acción es ciertamente antiguo. Así ocurre también con el término de Service Learning, visible desde 1967 en EEUU.

Este aprendizaje por proyectos con un fin solidario cada vez está siendo más valorado como herramienta en el desarrollo de los movimientos actuales mencionados sobre SmartCities, Smart-Personas, ciudanía concienciada y comprometida. Tanto es así que entre los primeros premiados en 2016 de la red de Ciudades Educadoras no sólo estaba una iniciativa de Finlandia y otra de Corea, sino también El aprendizaje servicio como herramienta de convivencia y cohesión social de L'Hospitalet de Llobregat (España) (AICE, 2018).

¿Por qué aprendizaje y servicio solidario (AySS) para alcanzar Ciudades Amigables? Son precisamente las dificultades en su contagio (una revolución pedagógica necesaria, como defiende Roser Batlle) las evidencias de su pertinencia. Aquí algunas cuestiones que podrían seguir sosteniendo algunas resistencias a este tipo de innovación:

- En el ámbito educativo: ¿Ciencia versus solidaridad? ¿Ética vs. tecnología? ¿Elearning como fin o como medio? ¿También la interdisciplinariedad se percibe como una amenaza? ¿Todos aprenden con el aprendizaje basado en proyectos?

- En el ámbito social, ¿Se

García, A. ; Villa, A.; Mugarra, A. (2018). Procesos de investigación-acción en aprendizaje y servicio solidario: percepciones de los protagonistas pedagógicos en el proyecto Hiri Lagunkoiak. RIDAS, Revista Iberoamericana de Aprendizaje Servicio, 5, 62-86. DOI10.1344/RIDAS2018.5.5 
confunde estar concienciada con estar comprometida? ¿Nos fiamos de la juventud para desempeñar competentemente acciones con impacto social? ¿Están y estamos preparados?

Al tratar de convertir estos problemas en retos pareciera que estuviéramos hablando de ética-ficción (Cortina, 1999). Sin embargo, cuando ofrecemos oportunidades de investigaractuar ¿Qué dicen los protagonistas pedagógicos?, ¿Qué hacen?, ¿Cómo se transforman y ayudan a transformar? En este artículo aportamos un ejemplo al respecto desde un proyecto para fomentar la conciencia en accesibilidad urbana, en concreto desde la mirada de movilidad reducida, en el que adolescentes y personas con diversidad funcional mapean su entorno (compartiéndolo en OpenStreetMap) y plantean propuestas de mejora (mediante un informe de accesibilidad) (Zerbikas, 2016, $1)$.

Hiri Lagunkoiak (Ciudades Amigables en euskera) comenzó en 2012, gracias al encuentro estatal de aprendizaje servicio en Barcelona. Desde entonces, continúa construyéndose en espiral. Así hemos tratado de compartirlo en algunas de las publicaciones al respecto:

- Propuesta de investigación- acción universitaria desde ingeniería, derecho y educación (Mugarra, Borges y García-Pérez, 2015).

- Estudio pre-piloto evidenciando las necesidades en la sistematización de la reflexión-acción (GarcíaPérez et al, 2017) .

- Resultados de las primeras 100 personas (García-Pérez et al, 2016).

- Unidades didácticas en abierto y empleo de la herramienta de software libre junto a algunos resultados cuantitativos de las 1000 personas participantes (Gómez Goiri et al, 2017).

En este artículo nos centramos en la investigación acción realizada por los protagonistas pedagógicos (adolescentescomunidad) y presentamos las evidencias del proceso a través de nuestro análisis cualitativo de sus percepciones.

\section{La filosofía desde la que partimos}

¿Qué es aprendizaje y servicio solidario? Si bien desde la aparición del concepto en 1967 en EEUU han surgido cientos de definiciones (Villa, 2013), lo retomamos aquí desde su interpretación como aprendizaje por proyectos con un fin solidario. 
La paradoja de conceptos como aprendizaje servicio crítico reside en la siguiente cuestión: ¿Por qué hemos llegado a tener que explicitarlo? ¿Qué está ocurriendo con la reflexiónacción en AySS? ¿Demasiado guiada y se pierde la esencia del protagonismo pedagógico para la justicia social? ¿Por qué no se refuerza la investigaciónacción de los protagonistas pedagógicos? Este es uno de los motivos por los que reivindicar la metodología de AySS desde el enfoque de innovación social universitaria responsable: por su bidireccionalidad con la comunidad, la evaluación compartida de impactos y los retos de acompañamiento universitario a la comunidad en Ciencia Ciudadana (GarcíaPérez, Mugarra y Villa, 2016).

\subsection{Movimientos de Ciudades}

Amigables y aprendizaje y servicio solidario

Los movimientos actuales de Ciudades Amigables, especialmente desde 2012, han tenido una creciente expansión internacional. Esta amigabilidad de los entornos puede concretarse desde diversos criterios e iniciativas, aquí algunos ejemplos diferenciadores:

- Ciudades Amigables. Una de sus acepciones más conocidas es la promovida por la Organización Mundial de la Salud al respecto de la búsqueda de indicadores de amigabilidad de las ciudades con las personas mayores (Buffel, Phillipson y Scharf, 2012).

- Ciudades Accesibles. En este caso se autodefine en términos de inclusión desde la diversidad funcional $y$, en concreto, desde la accesibilidad cognitiva, para promover su vida independiente. Como ejemplo, La Ciudad Accesible (Brusilovsky, 2016).

- Ciudades Educadoras. Aunque son famosas iniciativas como las de $L a$ ciudad de los niños (Tonucci, 1991-2015), el movimiento en general no se dirige exclusivamente a infancia y juventud, sino a la mejora de calidad de vida de cada persona ciudadana involucrada en actividades compartidas desde una perspectiva intergeneracional en aprendizaje a lo largo de la vida.

¿Dónde encaja AySS en todo esto? Retomando la cita de Laura Campo en la introducción, entendemos también que el aprendizaje servicio es una propuesta educativa que toma sentido en un territorio donde todos sus miembros $y$ entidades tienen o pueden tener un papel educativo $(2015,162)$. 
Ciudades Amigables para todas las personas: investigaciónacción en accesibilidad urbana a través de OpenStreetMap es un ejemplo de proyecto en el que confluyen ambos movimientos, así como todos los colectivos y objetivos descritos en dichas concepciones de Ciudades Amigables/Accesibles/Educador as. De hecho, las personas mayores activas y solidarias (Mendia, 2017) han sido y son parte fundamental de esta iniciativa intergeneracional: como expertos por experiencia directa, al haber tenido algún tipo de movilidad reducida $y / 0$ al haber sido acompañantes de personas con movilidad reducida.

Desde este ejemplo de proyecto de AySS en Ciudades Amigables, y dadas las publicaciones citadas justificando su fundamentación desde el modelo ISUR, software libre y Ciencia Ciudadana, en este artículo nos centramos en las percepciones cognitivas, emocionales y morales de los participantes, en cuanto a los Derechos Humanos especialmente de Accesibilidad y Ciencia, cuyo ingrediente común es la universalidad de la participación (figura 1).
Figura 1. Relación entre AySS y Ciudades Amigables: un ejemplo de proyecto para cambiar percepciones desde la investigación-acción en Accesibilidad Urbana.

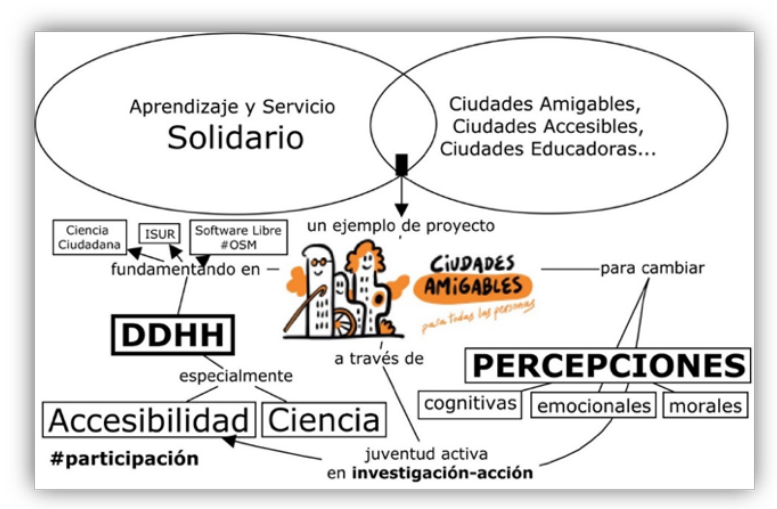

Fuente: Elaboración propia.

\subsection{Percepciones de}

Investigación-Acción en este proyecto de AySS y Ciudades Amigables

La psicología social nos ofrece dos elementos sobre el que pivotar nuestro análisis: desde las percepciones (Bandura, 1987) y desde la investigaciónacción participativa (Fals Bordá, 2005). El significado otorgado por cada persona a determinadas conductas y adaptaciones en contextos es esencial en los procesos de investigación-acción:

- Kurt Lewin (1946) destaca la pertinencia de este tipo de proceso investigador orientado al cambio social a través de toma de decisiones con relaciones intergrupales. Cualquier investigación- 
acción precisa (Karlsen y Larrea, 2015) participación. Pasar del para al con los stakeholders.

- Investigación. A través de métodos contrastados en la recogida y análisis del data y/o evidencias del proceso.

- Acción. Interacciones orientadas al cambio social.

La perspectiva crítico-social de la investigación-acción favorece su aplicabilidad también a la intervención educativa (Carr y Kemmis, 1986), mejorandoaprendiendo mediante los cambios generados, participando en espiral de ciclos, colaborando en responsabilidades y creando comunidades autocríticas en cada fase.

Así, ofrecemos un breve marco teórico sobre las percepciones en cuanto a los procesos de investigación-acción desde los tres componentes indispensables de un proyecto de ApS: necesidad, aprendizaje y servicio. En concreto, dotándoles de contenido con el proyecto Hiri Lagunkoiak y trabajando la investigaciónacción a partir de las fases identificadas de reflexión-acción (figura 2).
Figura 2. Fases de reflexión-acción en el proyecto.

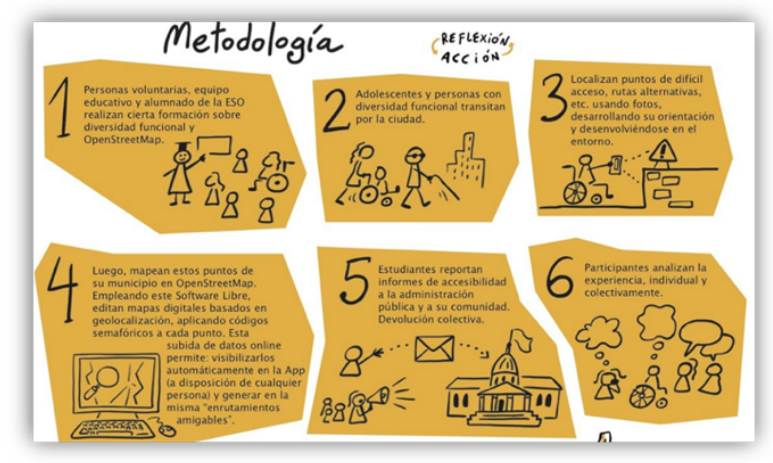

Fuente: ilustración realizada por Pernan Goñi y contenido por Zerbikas (2016).

\subsubsection{Necesidad social: percepción de la Accesibilidad Urbana como Derecho.}

La discapacidad es un concepto que evoluciona y que resulta de la interacción entre las personas con deficiencias y las barreras debidas a la actitud y al entorno que evitan su participación plena y efectiva en la sociedad, en igualdad de condiciones con las demás. ONU, 2006, 2.

Este énfasis en la participación es la clave común con otras comunicaciones como las de la Organización Mundial de la Salud. En este proyecto nos hacemos eco especialmente de dos derechos universales:

- Accesibilidad. La Convención sobre los Derechos de las Personas con Discapacidad en su artículo 9 describe este derecho, el cual deben hacer suyo en su legislación y cumplimiento los Estados firmantes (ONU, 2006). Por su parte, el diseño universal 
está todavía en desarrollo y necesitará de más investigación para determinar su efectividad (Johnstone et al, 2008, 35).

- Ciencia. La Declaración Universal de los Derechos Humanos de 1948, dice en el artículo 27: Toda persona tiene derecho a tomar parte libremente en la vida cultural de la comunidad, a gozar de las artes y a participar en el progreso científico y en los beneficios que de él resulten. Ello es independiente de la edad, y/o de la situación de discapacidad de cada persona.

La participación de personas con movilidad reducida en calidad de coinvestigadores en este proyecto no sólo incrementa su consecución en ambos derechos, también reivindica el concepto de expertos por experiencia iniciado en Salud Mental (McLaughlin, 2009).

Así, la necesidad social de Hiri Lagunkoiak se centra en la Accesibilidad Urbana como derecho universal, no cuestionable. Y, a la vez, parte de la participación cívica como derecho, especialmente vulnerado en jóvenes y personas con movilidad reducida. Los cambios en la percepción de este derecho universal (y de cualquier necesidad) están inevitablemente unidos no sólo a la visión del entorno (a través de evidencias que recogen $y$ analizan ellos mismos), sino también a sus cambios de visión con respecto a diversos colectivos, inferidas desde las relaciones con personas concretas, con espacios de encuentro común generando acercamiento con nuevas emociones/sentimientos y su repercusión en valores compartidos desde las componentes interrelacionadas de aprendizaje (competencial) y servicio (solidario).

\subsubsection{Aprendizaje competencial:} vocaciones científico-solidarias e inteligencia interpersonal.

En este tipo de proyectos el conocimiento científico da respuesta y se pone al servicio de una necesidad social sentida, se utiliza para salir de uno mismo, solidarizarse, ayudar a otros... En este sentido, conocer y valorizar no sólo se vuelven elementos indisociables, sino que, al unirse, multiplican el valor pedagógico de esta actividad.

Rubio, 2009, 96.

AySS promueve la interacción entre el conocimiento científico y la ética, y ello implica también la pertinencia del entrenamiento en el método científico para solidarizarse, para conocer y valorizar. Quizá, en dicha cita de Laura Rubio sería interesante incluir el sentir y el sentirse como clave para desarrollar los valores morales, más allá de la discusión de dilemas propuestas por referentes como Kohlberg (Hersh, Reimer y Paolitto, 1984). En AySS es esencial el valor añadido de los valores, lo citamos aquí desde su conjugación junto a otros tres elementos en la formación de la inteligencia interpersonal (aprender a pensar, educación emocional y desarrollo de habilidades sociales): 
El desarrollo moral y ético de manera que el alumno/a pase de una moral heterónoma, dependiente de otros, a una moral autónoma en la que acepte y se comporte conforme a principios morales universalizables, sin necesidad de recurrir a un control externo para regular su conducta (Uruñuela, 2016, 184-185).

Podríamos asociar este modelo de inteligencia personal con la categorización de percepciones cognitivas, emocionales y morales, en conexión con el aprendizaje por competencias y su interacción con modelos de análisis en AySS como el de Miquel Martínez (ApS en Cataluña y España en general) o el de Chantal Jouannet ( $A+S$ en Chile).

Las competencias abordan contenidos del currículum, habilidades psicosociales y educación en valores. Esta tríada de conocimientos-habilidades-valores coincide en trasfondo con las dimensiones de AySS: académica, comunitaria y formación en valores (Jouannet et al, 2015); también en cuanto a la dimensión ética en formación deontológica, ciudadana y humana (Martínez, 2010); y a la pertinencia del AySS como estrategia para alcanzar el desarrollo y evaluación de competencias a través del enfoque de Innovación Social Universitaria Responsable (Ugueño, Villar y Uribe, 2018).

Por otro lado, el AySS como aprendizaje por proyectos con fin solidario es también un aprendizaje para todos. De hecho, Andrew Furco (2015) resalta que incrementa especialmente el rendimiento del alumnado en riesgo de fracaso (escolar, universitario, etc.), ya que existe una mayor motivación: asociada al sentimiento de pertenencia en un grupo y a la percepción de utilidad (de lo aprendido, de lo transferido a la realidad y de sí mismos). También Mel Ainscow destaca el desarrollo de valores inclusivos en acción (Booth y Ainscow, 2015, 1), esta educación inclusiva con los ejes de presenciaparticipación-aprendizaje puede impulsarse desde el AySS. Este proyecto de Hiri Lagunkoiak es un ejemplo de ello (García-Pérez, 2016).

Con todo ello, se trata de aprender para crear a lo largo de su vida redes emocionales, redes sociales y redes profesionales estructuradas, pero eso solo se puede construir a través de pedir ayuda y dar ayuda (Toro, 2014).

\subsubsection{Servicio solidario:justícia y compromiso social transformador}

Con la solidaridad conviene llevar cuidado, ya que sólo es un valor moral cuando no es solidaridad grupal, alérgica a la universalidad, sino solidaridad universal, es decir, cuando las personas actúan pensando no sólo en el interés particular de los miembros de un grupo, sino también de todos los afectados por las acciones del grupo.

Cortina, 1997, 244. 
La manera de llevar los

conocimientos, las emociones y los

valores a la acción va a determinar

el sentido de desempeño en la

reflexión y en la acción:

¿oportunista?, ¿caritativa?, ¿asistencialista?, ¿transaccional?

¿prosocial?, ¿solidaria?, ¿transformacional? Trascender la solidaridad más allá del beneficio de uno mismo o incluso del propio grupo implica comprometerse con causas que en principio no fueron consideradas como propias.

Esta relación personal temprana con la otredad construye su capacidad de empatía y compasión y da como resultado una visión más inclusiva de los demás (Cipolle, 2010, 29); de hecho, cambian su opinión sobre quién es uno de nosotros a través del compromiso constructivo con la otredad (34). Sin embargo, esta ética del cuidado precisa de algunas consideraciones, desde la perspectiva comunitaria de Ciudad/Comunidad Cuidadora que comienza en la relación entre dos personas: la compasión es necesaria, pero no suficiente pues es necesario que se realicen acciones coherentes con el valor absoluto de la humanidad (Ramos Pozón, 2011, 120).

Cipolle (2010) identifica tres etapas en el desarrollo de la conciencia crítica desde AySS: caridad, cuidado y justicia social. Ese cuidado de las relaciones forma así parte del camino hacia la justicia social, pero es preciso algo más. La noción de justicia implica la comprensión de un problema en el sistema que trasciende más allá de la situación de una persona o de un colectivo, a un cambio sistémico con compromiso comunitario. Marina habla incluso de inteligencia comunitaria, pues de nada vale que tengamos derechos si la sociedad no nos ayuda a realizarlos, resaltando la compasión, el respeto y la indignación ante la injusticia (2013, $6)$.

Cortina (1997) enfatiza la justicia como trasfondo imprescindible en la educación de los principales valores cívicos: libertad (como participación, independencia y autonomía), igualdad, respeto activo, solidaridad y diálogo. Volvemos de nuevo a precisar de la interrelación del aprendizaje para mejorar el servicio, y viceversa. Esta retroalimentación depende a su vez de que ese otro con/para quien trabajamos sea bien reconocido (Puig y Bär Kwast, 2016), de cómo se construyan la reciprocidad y las relaciones transformacionales (Clayton et al, 2010).

Así, el desempeño y análisis del servicio solidario en Hiri Lagunkoiak, como en cualquier otro proyecto de AySS, requiere una triple mirada de sus transformaciones comunitarias, como ciudadanos comprometidos que forman parte de una comunidad: en sí mismos, en otras personas y en el entorno; a través del proceso y de los resultados del servicio.

\section{Metodología}

En la iniciativa Hiri Lagunkoiak 
coexisten diferentes tipos de investigación-acción: la realizada por el equipo universitario (acompañando en el entrenamiento de Ciencia Ciudadana a stakeholders) y la realizada por los protagonistas pedagógicos, siendo esta segunda desde la que realizamos nuestra contribución.

Ofrecemos un análisis cualitativo, dados los resultados de un cuestionario aplicado tras cada experiencia de investigación-acción realizada por estudiantes de Educación Secundaria Obligatoria. El motivo de selección de este colectivo alude a que, para contribuir a la resolución de la necesidad social (en este caso el fomento en conciencia cívica sobre accesibilidad urbana) es preciso contar con todos los miembros de la comunidad. Su investigación-acción ha implicado diversas fases:

1. Diagnóstico de la situación. El conocimiento les permite capacitarse y tener posibilidad de compartir sus impresiones (Park, 2006). Este acercamiento de los estudiantes a un problema real se realiza, en el caso de Hiri Lagunkoiak, a través de seminarios de sensibilización sobre accesibilidad.

2. Más etapas de apertura a otros puntos de vista (Martí, Montañés y Rodríguez-Villasante, 2002). En Hiri Lagunkoiak, esta apertura extra se ha realizado a través de mapeos urbanos junto a personas con movilidad reducida. No sólo han recogido datos, también los han compartido con la comunidad local e internacional en OpenStreetMap.

3. Procesos de negociación (Wilson, 2000), enriqueciendo la visión primigenia y elevándola a una más crítica y heterogénea. En Hiri Lagunkoiak han negociado en los grupos de discusión y elaborando informes de accesibilidad (entre diversas asignaturas y/o grupos-clase).

4. Evaluación y cierre, replanteando su propia concientización y la generada en otras personas a través de sus niveles de compromiso (Freire, 1978). La gestión por su parte de los actos de devolución de resultados y celebración con la comunidad han servido a su vez como toma de conciencia de la prospectiva y de las limitaciones de su investigación-acción.

El cuestionario consta de 16 preguntas, diseñadas para entrenar su reflexión individual al respecto de sus vivencias hasta el momento (mapeo y publicación de sus propios datos en abierto) y para fomentar así su participación en los grupos de discusión posteriores, orientados a explicitar sus cambios de percepción y a que generen propuestasproductos con los que realizar un efecto multiplicador. Este cuestionario se construyó en 2015 junto a FEKOOR (Federación Coordinadora de Personas con Discapacidad Física de Bizkaia), el claustro de profesorado del colegio Santa Maria, investigadores de tres facultades de la Universidad de Deusto y con la coordinación de la Fundación Zerbikas (Centro 
Promotor de Aprendizaje y Servicio Solidario en el País Vasco). Como herramienta de investigación, se ha asegurado el anonimato en la participación y su replicabilidad (Morales, 2011).

Tras una primera lectura de los primeros 100 resultados con inferencia inductiva, se ha analizado el contenido a partir de las categorías generadas tras la relectura y el primer esquema generado para los grupos de discusión, a repetirse con adecuaciones en cada uno de los mismos, tanto para los grupos en castellano como en euskera (Ruíz Olabuénaga, 2012).

Si bien en Hiri Lagunkoiak han participado 1000 personas entre 2015 y 2017 , ascendiendo a 800 el alumnado de 11 institutos de la Comunidad Autónoma del País Vasco (España), para este estudio únicamente se han analizado los resultados del cuestionario de intervención cumplimentado por 382 adolescentes (entre 12 y 18 años), en concreto de los 6 colegios y/o institutos que han respondido en castellano: Santa Maria (Portugalete); Tartanga (Erandio); Colegio Europa (Getxo); Madre de Dios, Ángeles Custodios y Centro Formativo Otxarkoaga (Bilbao).

Los instrumentos de investigación y datos recogidos (propuesta de modelo, herramienta de enrutamientos, vídeos, informes y otros productos generados) están disponibles en la web del proyecto (ciudadesamigables.org), en coherencia con el espíritu Open

Access que lo ha hecho posible.

\section{Resultados}

Con todo ello, aportamos aquí una síntesis del relato de los participantes sobres sus percepciones cognitivas, emocionales y morales al respecto de la necesidad atendida, el aprendizaje obtenido y el servicio realizado; con nuestro análisis desde diferentes miradas en cada dimensión (tabla 1).

Tabla 1. Análisis de las percepciones en el proyecto Hiri Lagunkoiak

\begin{tabular}{|l|l|l|l|}
\hline & $\begin{array}{l}\text { NECESIDAD } \\
\text { Social }\end{array}$ & $\begin{array}{l}\text { APRENDIZAJE } \\
\text { competencial }\end{array}$ & $\begin{array}{l}\text { SERVICIO } \\
\text { solidario }\end{array}$ \\
\hline $\begin{array}{l}\text { Obje } \\
\text { tivos } \\
\text { iniciativa }\end{array}$ & $\begin{array}{l}\text { Accesibilidad } \\
\text { Urbana como } \\
\text { derecho }\end{array}$ & $\begin{array}{l}\text { Proceso científico } \\
\text { e inteligencia } \\
\text { interpersonal }\end{array}$ & $\begin{array}{l}\text { Ciudadanía } \\
\text { comprometida }\end{array}$ \\
\hline $\begin{array}{l}\text { Objetivos } \\
\text { de }\end{array}$ & $\begin{array}{l}\text { Triple miradisis } \\
\text { social: } \\
\text { (in)justicia, } \\
\text { inclusión y } \\
\text { compromiso }\end{array}$ & $\begin{array}{l}\text { Triple mirada de } \\
\text { su protagonismo } \\
\text { pedagógico: } \\
\text { conocimientos, } \\
\text { habilidades, } \\
\text { valores }\end{array}$ & $\begin{array}{l}\text { Triple mirada } \\
\text { de sus } \\
\text { transformacio } \\
\text { nes } \\
\text { comunitarias: } \\
\text { en sí mismos, } \\
\text { en otras } \\
\text { personas y en } \\
\text { el entorno }\end{array}$ \\
\hline
\end{tabular}

Fuente: elaboración propia

\subsection{Percepción de la necesidad social}

La Accesibilidad Urbana como Derecho Universal se ha trabajado desde los cambios de percepción sobre el entorno (a través de evidencias recogidas por las y los participantes) y sobre las personas (a través de experiencias de encuentro en los mapeos, seminarios, talleres y celebraciones, como parte de su investigaciónacción compartida).

Para empezar, y por iniciativa de 
FEKOOR (a replicar en el resto de los seminarios con otras entidades como BENE, IGON, Elkartu), se invita al alumnado a describir con 3 palabras su concepción de la discapacidad. Entre las evidencias, predominan percepciones negativas como problema, dificultades, minusvalía, deficiencia, enfermedad; y se identifica como elemento diferenciador la silla de ruedas (figura 3).

Figura 3. Nube de palabras sobre discapacidad, previa al inicio del proyecto.

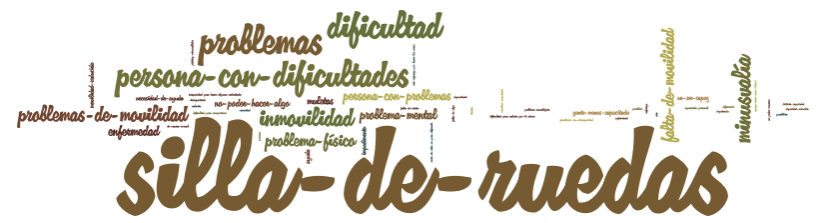

Fuente: elaboración propia

Figura 4. Nube de palabras sobre discapacidad, durante el desarrollo del proyecto.

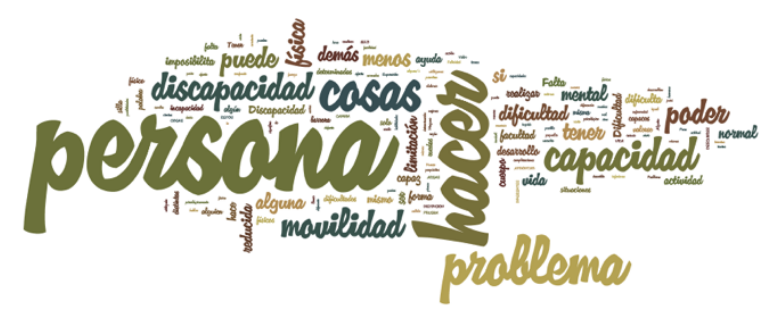

Fuente: elaboración propia.

En cambio, tras la experiencia de mapeo en grupos mixtos con las personas con movilidad reducida, su redefinición de discapacidad dista de su pre-concebida. Si bien la indicación de tres palabras sigue siendo la misma, esta vez contestan con oraciones elaboradas y subordinadas a determinados matices. Entre las evidencias encontrades (figura 4), se dan concepciones proactivas como reto, apoyos, diversidad, personas, situaciones. Perciben la complejidad de definir discapacidad, encontrando similitudes con lo que ocurriría con cualquier persona en términos de diversidad funcional:

Que no puede o tiene dificultad para hacer alguna/s cosas.

Que no tiene facilidad para hacer las cosas sin ayuda.

Una persona que no tiene capacidad para hacer todo.

Discapacidad es, en mi opinión, una palabra mal utilizada para definirse a personas con una diferencia, ya sea física o mental, a las demás personas.

Necesita ayuda para superar algunas situaciones.

Me parece que una discapacidad es una dificultad, pero no una limitación que muchas veces se confunde, porque con propósitos, esfuerzo y actitud todo es posible.

Destacamos la respuesta de un/a adolescente que, además de ajustarse a las 3 palabras, genera con ellas una idea vital, discapacidad es una percepción social. Ello responde en términos inclusivos y de justicia social a la cuestión iniciada en los seminarios por Javi Cueva y Diego Lastra: ¿la

García, A. ; Villa, A.; Mugarra, A. (2018). Procesos de investigación-acción en aprendizaje y servicio solidario: percepciones de los protagonistas pedagógicos en el proyecto Hiri Lagunkoiak. RIDAS, Revista Iberoamericana de Aprendizaje Servicio, 5, 62-86. DOI10.1344/RIDAS2018.5.5 
persona tiene discapacidad o es la sociedad quien discapacita?

Aún así, continúan existiendo (en las respuestas a esta pregunta y al resto sobre descripciones de la experiencia) cierta prevalencia de conceptos previos o incluso sentimientos como la pena, que nos instan a retomar la reflexión en directo de la manera más participativa posible.

Se realiza en cada caso también una devolución de resultados cuantitativos. En estas sesiones, el alumnado visibiliza en la plataforma los datos que han recogido y aporta un primer análisis que ha coincidido en todos los casos mapeados: una alta prevalencia de líneas (aceras) accesibles, relativas al ámbito de lo público; y una alta prevalencia de puntos (portales, comercios) no accesibles o accesibles sólo con ayuda, relativos al ámbito de lo privado. Ante ello, una de las primeras participantes expresó que Estamos arruinando a los bares, poniendo de manifiesto un tema muy relevante: la tensión entre lo público-privado. Ello introduce la cuestión desde la perspectiva de compromiso: ¿quién es responsable en ello y quién debe solucionarlo? ¿Cómo?

\subsection{Percepción del aprendizaje adquirido}

El alumnado menciona aprendizajes competenciales que consideran haber adquirido/incrementado gracias a Hiri Lagunkoiak, en términos de:
- Conocimientos (conceptos sobre accesibilidad de manera interdisciplinar; ciencia; ética, comunicación, e-learning...).

Una buena manera de conocer la ciudad en la que vivimos.

Han conseguido enseñarnos de manera divertida en la que podíamos participar todos.

Me gustaría seguir con el proyecto en lengua, para hacer más fácil el lenguaje (de toda esta información) para niños pequeños.

- Habilidades (incluido el trabajo en equipo; y cambio de mirada ética, autonomía y emprendimiento científicotecnológico; desempeño de proceso científico).

(Trabajar) en equipo mejor, todos aportamos, +rápido, +a gusto distribuyéndose las tareas.

Incluso habilidades a mejorar:

En el grupo, el sentido de la orientación brillaba por su ausencia, por lo tanto nos llevaba más tiempo mapear.

- Valores (prosocialidad, compañerismo, compromiso, solidaridad, alteridad, reconocimiento del otro), incluida la orientación a la democratización del conocimiento y la cooperación altruista desde el anonimato. 
Me parece que un mapa en el que cualquiera pueda editar $y$ contribuir es útil, sobre todo con toda la información que se puede añadir.

Además, el propio alumnado destaca su aprendizaje y atribución de valor a la experiencia especialmente desde:

1. El acompañamiento de personas con movilidad reducida, como expertos por experiencia, en los grupos mixtos de mapeo. Es significativo que aporten nombres concretos de las personas que les acompañaban (la persona más allá de su discapacidad) y que algunas personas expliciten un aprendizaje vital.

Nos dimos cuenta de que algunas veces les dejábamos de lado (de la toma de decisiones), sobre todo al principio.

Otras personas ni siquiera contestaron cuando se incorporó esta pregunta en la segunda edición y a otros les parecía obvio.

Claro, para eso nos acompañaron.

\section{Como si fueran nuestros} profesores.

En su mayoría, explicitan que les gustaría repetir la experiencia. Estas personas voluntarias les han ayudado a cambiar su percepción del entorno (antes no percibían las barreras de movilidad) y de la necesidad de los apoyos en la vida cotidiana de cualquier persona.

Antes pensaba que había una gran cantidad de actividades que no podían hacer, pero ahora me he dado cuenta de que realmente sí pueden. Lo único que necesitan es una adaptación de la actividad o algo que les ayude a realizarla.

Antes creía que eran inválidas $y$ ahora veo que nos pueden enseñar mucho; Nos ayudaron mucho y nos enseñaron en lo que debíamos fijarnos.

En general, valoran muy positivamente la experiencia de estar con.

2. El uso de la silla de ruedas en la calle. En este sentido ha habido una variedad muy dispersa de descripciones. Desde quienes dicen igual que siempre, pasando por aquellos que llegan a explicitar que se han sentido (peor) sólo físicamente, hasta quienes admiten haberse sentido

Observada y dependiente.

Débil, vulnerable.

Al principio me lo tomé como un experimento divertido, pero al de un rato tenía muchas ganas de levantarme y andar por mi cuenta, ya que me cansé de depender de 
otra persona.

Sinceramente, me lo tomé con humor, aunque comprendo que no les haga gracia a las personas que realmente lo padezcan. Creo que es fundamental tener esa actitud para adaptarse más rápido a esta sociedad.

Era difícil pasar por algunos tramos con la silla de Juan.

En ocasiones percibieron actitudes positivas de los viandantes y en otras todo lo contrario, desde la condescendencia al rechazo.

Cuando fuimos ayer con las personas en silla de ruedas, la gente te miraba raro, como si fueras menos que ellos $o$ así. Y muchas veces no te dejaban espacio para pasar.

Ambas componentes han mejorado la calidad de los datos recogidos sobre el entorno y su cambio de percepción sobre las personas acompañantes y acompañadas, imprescindible para comprometerse en la promoción de la accesibilidad desde una perspectiva prosocial.

\subsection{Percepción del servicio realizado}

El alumnado percibe en diferentes grados la atribución de valor al servicio realizado desde sus dos componentes:

- Proceso. Experiencia compartida como investigadores junto a personas con movilidad reducida y resto de comunidad educativa.

Gracias por esta experiencia. Ha estado muy bien porque además de conocer a otras personas y pasarlo bien, se aprende bastante y estas ayudando para algo que es útil y necesario.

Convivir para ayudar a otras personas te une.

- Resultados. Datos en OpenStreetMap, herramienta de enrutamientos, informes de accesibilidad (posteriores a estas respuestas del cuestionario).

No me gusta que una persona tenga que entrar al colegio por donde entra la comida o que tenga que subir la acera por la rampa de un garaje.

Quiero mejorar y completar el mapa.

4.4. Interacción de las percepciones de necesidad, de aprendizaje y de servicio

¿La compasión es necesaria? ¿Y suficiente? Lo hemos planteado en todos los grupos de discusión de Hiri Lagunkoiak, suscitada por lo analizado en cada grupo de cuestionarios de intervención tras los mapeos. En la respuesta se combinan aspectos mencionados en la necesidad, en el aprendizaje y en el servicio.

En las respuestas de los cuestionarios, interpretamos un 
continuum entre diferentes

emociones suscitadas, que a su vez tienen relación con la comprensión de la necesidad social (en términos de problema individual o de reto comunitario) y con los itinerarios derivados en la orientación a la acción, promocionando unos determinados valores. Algunos ejemplos:

\section{- Indiferencia}

\section{No me afecta.}

Este rechazo social tiene su motivación generalmente en el desconocimiento por inexperiencia, por no haber convivido con personas afectadas ni haber experimentado en primera persona una situación al respecto. Sin embargo, nos hemos encontrado con jóvenes que lo han manifestado teniendo a su lado a compañeros de aula con movilidad reducida permanente, por ejemplo; en ese caso el problema de empatía es alarmante y ese rechazo social pueden provocar situaciones de violencia por acción o por omisión.

\section{- Tristeza}

Me dan pena, pobrecillos.

Estos sentimientos en ocasiones están asociados a una actitud pasiva o, aún llegando a estar orientados a la acción, caritativa: ayudar a los necesitados (un término que no tiene por qué ser desfavorable si va acompañado posteriormente de reflexión crítica sobre las relaciones establecidas: ¿sólo se ayuda en una dirección? ¿somos salvadores?). En todo caso, perciben que existe un problema, aunque lo vinculen a una determinada persona/colectivo.

- Sentir(se) conmovido. Ante la situación en la que se encuentra la persona y/o colectivo, con conciencia de aspectos positivos y negativos en su recorrido. En la orientación a la acción:

Antes solo me preocupaba de que yo pudiera pasar, pero ahora también pienso a ver si las personas con discapacidad pueden acceder a los sitios que yo voy. Además de la alteridad, aparecen otros valores como empatía y prudencia.

- Compasión. Reconocimiento (empiezan por citar el nombre de la persona con discapacidad).

\section{Ayudar para facilitarles la} vida a otros.

Al ponerte en su lugar te das cuenta de que es realmente importante de que tengan la facilidad de moverse independientemente.

Percepción de que el problema no concierne solo a la persona con discapacidad y/o movilidad reducida, si no que existen causas propiciadas por el sistema. Entre los valores destacan el valor de libertadindependencia, respeto, 
dignidad, inclusión y diálogo.

\section{- Admiración}

Antes sentía pena, ahora les admiro.

Me gustaría seguir hablando con ellos, sobre sus experiencias, sentimientos.

En la orientación a la acción, relación de reciprocidad.

No siento pena (sin ánimo de ofender) porque implica cierto grado de superioridad. EI trato debería de ser de igual a igual.

Desde este reconocimiento, se percibe que pueden existir soluciones, aunque no se identifiquen responsables en el cambio. Otros valores vinculados a la reciprocidad son la confianza y gratitud.

- Indignados

Ahora solo me fijo en si los accesos a portales $y$ comercios están bien adaptados, me sorprende que haya pocos.

Te das cuenta de que tu barrio no está tan bien como creías y se puede cambiar. Es una primera etapa necesaria (pero no suficiente) para percibir que existen soluciones con las que comprometernos. Destaca el valor de justicia.

- Convencimiento de que es necesario un cambio social, ante los sentimientos provocados por la exploración del entorno: sorprendido, impactado, impresionada, impotente, frustrante... Hay un punto de diferencia entre el convencimiento de que es necesario cambiarlo (sin precisar quiénes, quizá es únicamente la administración):
El tiempo es necesario para transformar (...) Hace 20 años nadie pensaba en rampas, pero ahora las hay. Se necesitan más, sí, pero el propietario de una tienda seguramente no quiera cambiar un escalón solo por una persona discapacitada que pueda ir al año. El ayuntamiento es quien debería poner accesible las aceras y centros públicos.

$Y$ el convencimiento de que todos deberíamos estar implicados en dicho cambio:

Los accesos son necesarios en la vida cotidiana de una persona.

Valor de solidaridad asociado a sensibilidad, generosidad, responsabilidad o, incluso en ocasiones, amistad.

- Comprometidos y desde ahí implicados en la promoción del reto comunitario. Partir de la compasión hasta una implicación comprometida con-pasión. Valores de colaboración, 
participación, creatividad e incremento de sentimiento de pertenencia.

Antes no daba importancia a
que en Portugalete hubiera
muchas barreras para las
personas con dificultades para
moverse. Gracias a este
proyecto me he dado cuenta
de que es algo que
deberíamos cambiar porque
todos tenemos el derecho a
tener acceso a cualquier
portal, comercio....

Con todo ello, aportamos un gráfico de interacción entre la percepción de emociones, percepción de (in) justicia y compromiso comunitario (figura 5).

Figura 5. Interacción entre las transformaciones de emocionessentimientos, de la percepción de la (in)justicia social y de la orientación a la acción.

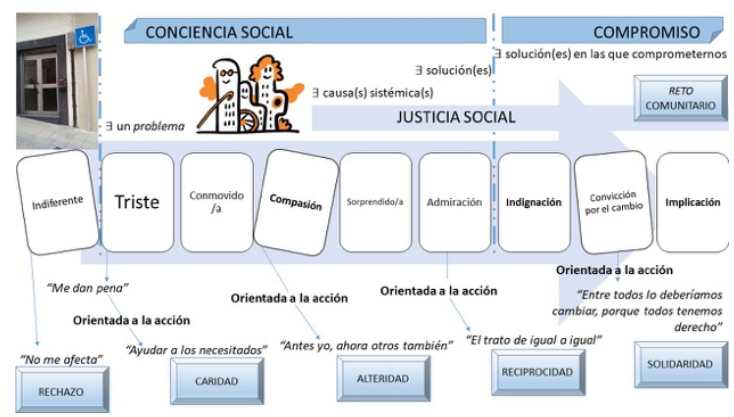

Fuente: elaboración propia

¿Qué entendemos exactamente por orientación a la acción? ¿Lo desarrollamos desde una perspectiva inclusiva? Ilustramos esta cuestión con ejemplos de desempeño en tres casos con tiempos y colegios diferentes:
1. Presencia. A pesar de haberse creado grupos mixtos para el mapeo de alumnado de Educación Secundaria Obligatoria con alumnado de Educación Especial, a estos últimos no se les había explicado la actividad, ni si quiera a sus educadores. Resultado contraproducente: no participaron, solo acudieron como turismo exótico, no se atribuyó sentido a la experiencia y expresaron sentirse frustrados.

2. Participación. Alumnado de Educación Especial sale a mapear en grupos mixtos con alumnado de ESO. Sí están implicados en el entrenamiento del mapeo el alumnado y el equipo educador de ambos colectivos. La experiencia de mapeo resulta muy enriquecedora: el alumnado de Educación Especial adquiere el rol de experto por experiencia, con relación y reconocimiento en doble vía con sus compañeros del aula ordinaria, y además los datos recogidos por estos grupos mixtos son más fiables.

3. Aprendizaje. Si bien en este caso el alumnado de Educación Especial no salió a mapear en grupos mixtos con alumnado de ESO, con la consecuente oportunidad perdida en términos de diversidad, sí que demostraron incrementar su aprendizaje a través de su aportación de investigaciónacción en esta iniciativa. No sólo recogieron datos de mapeo, también los subieron por sí mismos en un taller de 
OpenStreetMap e incluso

explicaron el procedimiento a sus educadoras.

Como consecuencia de estos procesos de reflexión orientados a la acción, aportamos algunos ejemplos de productos $\mathrm{y} / \mathrm{u}$ otras transformaciones (como resultado de sus niveles de implicación) realizados por los protagonistas pedagógicos: datos de accesibilidad compartidos en OpenStreetMap y enrutamientos en ciudadesamigables.org; informes de accesibilidad indoor ante sus institutos, creándose por ejemplo rampas de acceso desde la puerta principal; adaptación del tríptico en lectura fácil; informes de accesibilidad a sus representantes municipales, con repercusión en prensa y recepción de las autoridades, e ideas planteadas por alumnado con discapacidad, por ejemplo, para realizar rampas en sus talleres de carpintería a ofertar a comercios de su entorno.

Las sesiones de devolución de resultados, más allá de lo comprometido desde la ética de la investigación, propician a su vez más beneficios para la intervención, retomando procesos de reflexión individual desde el diálogo compartido y generando prospectiva. Entre otras aportaciones, el voluntariado con movilidad reducida $\mathrm{y} / \mathrm{o} \mathrm{P}+$ (personas mayores activas y solidarias), coincide en la necesidad percibida de adaptar la herramienta de enrutamiento de ciudadesamigables.org en términos de accesibilidad sensorial y cognitiva. Si bien es cierto que es mejorable el grado de autonomía en la comprensión de su uso, por el momento todos los grupos han conseguido con acompañamiento subir sus datos a OpenStreetMap y visualizarlos a través de talleres.

\section{Conclusiones}

\begin{abstract}
¿Pueden existir ciudades amigables/accesibles/educadoras sin personas accesibles? Si bien en esta iniciativa nos hemos centrado en la movilidad reducida para generar datos sistematizados, la amigabilidad de los entornos no puede reducirse a una perspectiva y excluir otras necesidades de accesibilidad como la cognitiva, desde donde se reivindica este concepto actual de personas accesibles (Cabezas, 2017). Se trata de aspirar a una accesibilidad alcanzada por aproximaciones sucesivas, en las que a la vez que los proyectos-iniciativas de base vayan reivindicando y divulgándose, también desde ahí se permeabilice en doble vía a la legislación y al proceso de institucionalización en cada entidad/grupo de personas.
\end{abstract}

La educación de dichas personas accesibles no es casual, y consideramos que AySS es una potencial herramienta para lograrlo, sumándose a iniciativas desarrolladas o por llegar. Destacamos para ello la dimensión de glo(c)alidad, con una perspectiva global sobre justicia social y una aplicación que dote de sentido en lo local; aprovechando recursos 
disponibles en el territorio cercano y por supuesto en el ámbito internacional, diversas combinaciones con lo online posibilitan encuentros por explorar (Strait y Nordyke, 2015).

Conscientes, implicados e indignados (...) con la necesidad de reaccionar (Hessel, 2011c, 9-10). ¿Primero la implicación para indignarse? ¿o indignarse para implicarse? Indignarse, comprometerse y actuar (Hessel, 2011a, 2011b, 2011c) son procesos retroalimentados por la reflexiónacción, y hemos visto su orden alterado en diferentes casos con Hiri Lagunkoiak. Hay que brindar a la juventud oportunidades de participación para que puedan percibir esa necesidad (de participación social) y enriquecerse colectivamente. Si nunca lo han experimentado, es menos probable que vayan a valorar la posibilidad de implicación/compromiso social en su futuro laboral/personal. El mismo está irremediablemente unido a las percepciones sobre sus vivencias, y desde ahí a sus emociones, reflexión vinculada a la experiencia. La reflexión-acción aporta contenido a la educación emocional; y la componente solidaria incluye la proyección en valores. En este sentido y en AySS, la tristeza como conclusión en nuestros educandos es un fracaso como educadores: no porque ese sentimiento no sea legítimo por sí mismo, sino porque su orientación a la acción puede ser contraproducente, por ejemplo, generando actitudes paternalistas.
Hiri Lagunkoiak nos ha permitido cuestionar los procesos de reflexiónacción y valorar sus posibilidades como investigadores en cada grupoclase. ¿Quiénes son realmente los protagonistas pedagógicos? ¿Cómo se evalúan sus transformaciones? Por supuesto, el estudio de las percepciones no es suficiente, pero sí necesario; así como también promocionar la investigación-ccción, crítica-empoderadora-creativatransformadora que contribuya en la construcción de una ciudadanía concienciada, comprometida, activa, colaborativa, competente y solidaria.

\section{Referencias bibliográficas}

\section{Asociación Internacional de Ciudades Educadoras (AICE) (2018). Recuperado de http://www.edcities.org/}

\section{Bandura, A. (1987). Pensamiento y acción. Fundamentos sociales. Barcelona: Martínez Roca.}

Booth, T. y Ainscow, M. (2015). Guía para la educación inclusiva.

Desarrollando el aprendizaje y la participación ciudadana en los centros escolares. Madrid: OEIFUHEM. Recuperado de

Brusilovsky Filer, B. L. (2016). Innovaciones en accesibilidad cognitiva. Entornos urbanos que hablan a las personas. Colección Democratizando la Accesibilidad, vol. 11. Granada: La Ciudad Accesible.

Buffel, T., Phillipson, C. y Scharf, T. (2012). Ageing in urban 
environments: Developing 'agefriendly'cities. Critical Social Policy, 32(4), 597-617.

Cabezas, D. (2017). Plan de accesibilidad cognitiva de Gorabide. I Congreso Estatal de Accesibilidad Cognitiva, Cáceres.

Campo, L. (2015). Para implantar y difundir el aprendizaje servicio conviene aplicar estrategias locales. En J.M. Puig (Coord.). 11 ideas clave, ¿Cómo realizar un proyecto de aprendizaje servicio? pp 161174. Barcelona: Graó.

Clayton, P. H., Bringle, R. G., Senor, B., Huq, J. y Morrison, M. (2010). Differentiating and Assessing Relationships in Service-Learning and Civic Engagement: Exploitative, Transactional, or Transformational. Michigan Journal of CommunityService Learning, 16(2), 5-22.

Cortina, A. (1997). Ciudadanos del mundo. Hacia una teoría de la ciudadanía. Madrid: Alianza.

Cortina, A. (1999). Los ciudadanos como protagonistas. Barcelona: Galaxia Gutenberg.

García-Pérez, A. (2016). Liderazgo prosocial del educador fomentando la inclusión: un proyecto sobre accesibilidad urbana a través de OpenStreetMap. XII Congreso Internacional y XXXIII Jornadas de Universidades y Educación Inclusiva. Albacete: Universidad de Castilla La Mancha.

García-Pérez, A., Mugarra, M. y
Villa, A. (2016). Innovación Social Universitaria como marco para la fundamentación, desarrollo y evaluación comunitaria de proyectos de Aprendizaje-Servicio. Educación y Diversidad, 10(2), 77-86.

García-Pérez, A., Pijoan, A., Borges, C.E., de Casas, S., Echaniz, A., Alonso, A. y Mugarra, A. (2016, septiembre). "Ciudades Amigables": combining service learning with citizen science to improve urban accessibility information on OpenStreetMap. 2016 Annual International Conference, Learning GIS. Londres: Royal Geographical Society.

García-Pérez, A., Pijoan, A., Borges, C.E., Alonso, A. y Mugarra, A. (2017). Consecución de la fase preparatoria de Ciudades Amigables para Todos: Investigación-Acción universitaria en Accesibilidad urbana a través de OpenStreetMap. En F. J. Carrillo-Rosúa, J. L. Arco-Tirado y F.D. Fernández-Martín (Eds.), Investigando en la mejora de la enseñanza universitaria a través del Aprendizaje Servicio, pp 429-435. Granada: Universidad de Granada. Disponible en http://editorial.ugr.es/pages/publica cionesenabierto/investigandolamejor a/!

Gómez Goiri, M., García-Pérez, A., Pijoan Lamas, A., Echaniz, A., Borges, C.E., Alonso-Vicario, A. y Mugarra Elorriaga, A. (2017). Hiri Lagunkoiak - Ciudades Amigables Friendly Cities 4All: Interdisciplinariedad y Compromiso sobre Accesibilidad Urbana en 
Secundaria. Libro de actas CIMIE17 de AMIE. Asociación Multidisciplinar de Investigación Educativa.

Disponible en

http://amieedu.org/actascimie17/w p-content/uploads/2016/06/269.pdf

Fals Borda, O. (2005). Participatory (Action) Research in Social Theory: Origins and Challenges. En P.

Reason y $\mathrm{H}$. Bradbury, Handbook of Action Research. Participative

Inquiry and Practice. pp. 27-37. Londres: SAGE.

Furco, A. (2015, mayo). A Status Report on the Impacts of ServiceLearning: A Review of the Research. Conferencia invitada. Bilbao: Universidad de Deusto y Fundación Zerbikas.

Hersh, R., Reimer, J. y Paolitto, D. (1984). El crecimiento moral: de Piaget a Kohlberg. Madrid: Narcea.

Hessel, S. (2011a). iIndignaos! Un alegato contra la indiferencia y a favor de la insurrección pacífica. Barcelona: Destino.

Hessel, S. (2011b). iComprometeos! Ya no basta con indignarse: conversaciones con Gilles Vanderpooten. Barcelona: Destino.

Hessel, S. (2011c). Es tiempo de acción. En R.M. Artal (Coord.). iReacciona! 10 razones por las que debes actuar frente a la crisis económica, política y social, pp. 910. Madrid: Santillana.

Johnstone, C. J., Thompson, S. J., Bottsford-Miller, N. A. y Thurlow, M. L. (2008). Universal Design and
Multimethod Approaches to Item Review. Educational Measurement: Issues \& Practice, 27(1), p. 25-36.

Jouannet, C., Montalva, J. T., Ponce, C., y Von Borries, V. (2015). Diseño de un modelo de institucionalización de la metodología de aprendizaje servicio en educación superior. RIDAS. Revista Iberoamericana de Aprendizaje Servicio, 1, 112-131.

Marina, J.A. (2013). Prólogo Aprendizaje-Servicio. En R. Batlle, El Aprendizaje-Servicio en España: el contagio de una revolución pedagógica necesaria, pp. 5-10. Madrid: PPC.

Martínez, M. (Ed.). (2010).

Aprendizaje servicio y responsabilidad social de las universidades. Barcelona: Octaedro.

McLaughlin, H. (2009). What's in a name:

'client','patient','customer','consumer' ,'expert by experience','service user'-what's next?. The British Journal of Social Work, 39(6), 11011117.

Mancisidor, M. (2017). El derecho humano a la ciencia: Un viejo derecho con un gran futuro. Anuario de Derechos Humanos, (13), 211221.

Mendía, R. (2017). Guía 7, Aprendizaje Servicio y $P+$. Personas Mayores Activas y Solidarias. Zerbikas Fundazioa y Hartu Emanak: Bilbao.

Morales, P. SJ (2011). Guía para construir cuestionarios y escalas de 
actitudes. Ciudad de Guatemala: CINDEG, Universidad Rafael Landívar.

Mugarra, A., Borges, C. E. y GarcíaPérez, A. (2015). Ciudades

Amigables para todos:

investigación-acción universitaria en accesibilidad urbana a través de OpenStreetMap. En P.

ARAMBURUZABALA, H. OPAZO y J. GARCÍA GUTIÉRREZ (Eds.), El Aprendizaje Servicio en las universidades: de la iniciativa individual al apoyo institucional, pp. 245-257. Madrid: UNED.

Soria, K.M. y Mitchell, T.D. (Eds.). (2016). Civic Engagement and Community Service at Research Universities. London: Palgrave Macmillan UK.

ONU (2006). Convención sobre los Derechos de las Personas con Discapacidad y Protocolo. Facultativo. Disponible en http://www.un.org/disabilities/docu ments/convention/convoptprot-s.pdf

Ramos Pozón, S. (2011). La ética del cuidado: valoración crítica y reformulación. Laguna: Revista de Filosofía, (29), 109-122.

Puig Rovira, J.M. y Bär Kwast, B. (2016). Reconocimiento y aprendizaje servicio. RIDAS, Revista Iberoamericana de Aprendizaje y Servicio, 2, 139-165.

Rubio, L. (2009). El aprendizaje en el aprendizaje servicio. En J.M. Puig (Coord.). Aprendizaje Servicio (ApS). Educación y compromiso cívico (pp. 91-106). Barcelona:
GRAÓ.

Ruíz Olabuénaga, J.I. (2012). Metodología de la investigación cualitativa (5 ed). Bilbao: Universidad de Deusto.

Strait, J., y Nordyke, K. (Eds.). (2015). EService-learning: Creating Experiential Learning and Civic Engagement Through Online and Hybrid Courses. Sterling: Stylus.

Tonucci, F. (1991-2015). La ciudad de los niños. Barcelona: GRAÓ.

Toro, B. (2014, agosto). El cuidado: el nuevo paradigm ético de la educación. Conferncia en XVII Seminario Internacional de Aprendizaje y Servicio Solidario. Buenos Aires: CLAYSS.

Ugueño, A., Villar, J. y Uribe, P. (2018). Aprendizaje Servicio (AS) como estrategia integral de evaluación en un modelo educativo basado en competencias. En A. Villa (Ed.), Tendencias actuales de las transformaciones de las universidades en una nueva sociedad digital. Vigo: FIIU.

Uruñuela, P. (2016). Trabajar la convivencia en los centros educativos. Una mirada al bosque de la convivencia. Madrid: Narcea.

Villa, A. et al. (2013). ISUR. Un modelo de evaluación de la Innovación Social Universiatria Responsable. Bilbao: Tuning.

Zerbikas Fundazioa (2016). Hiri Lagunkoiak - Ciudades Amigables Friendly Cities 4All. Bilbao: 
Zerbikas.

\author{
${ }^{i}$ Este proyecto ha recibido financiación de la \\ Fundación Española de Ciencia y Tecnología \\ (FECYT) y del Gobierno Vasco (beca \\ predoctoral), entre otros. Las entidades \\ participantes, financiadoras y premios \\ obtenidos aparecen en \\ ciudadesamigables.org
}

